\title{
ON VANISHING ALGEBRAS
}

\author{
BY TENG-SUN LIU ${ }^{1}$ \\ Communicated by I. J. Schoenberg, January 2, 1962
}

Let $G$ be a locally compact group with left invariant Haar measure $m$. For any measurable subset $S$ of $G$, define $L_{S}$ to be that subset of $L^{1}(G)$ consisting of all functions which vanish (a.e.) on the complement of $S$. When $L_{S}$ forms an algebra, we call it a vanishing algebra. It is known that when $S$ is a semigroup l.a.e. (i.e., there exists a semigroup $T$ in $G$ such that $S=T$ locally almost everywhere), $L_{S}$ is a vanishing algebra. The following theorem gives an answer to a problem formulated by A. Simon [2]:

THEOREM 1. Suppose $G$ is unimodular. If $L_{S}$ is a vanishing algebra and $S$ is contained in a $\sigma$-compact subset of $G$, then $S$ is a semigroup a.e.

Corollary 1. Suppose $G$ is compact. Then, if $L_{S}$ is a vanishing algebra, $S$ is a semigroup a.e.

Corollary 2. Suppose $G$ is abelian and generated by some compact neighborhood of the identity element of $G$. Then, if $L_{S}$ is a vanishing algebra, $S$ is a semigroup a.e.

The proof of Theorem 1 also gives the following more general and involved statement:

Theorem 2. Let $L_{S}$ be a vanishing algebra. Suppose there exists a directed set $\left\{U_{i}, i \in I\right\}$ of symmetric neighborhoods of the identity element $e$ with finite measures, having the property that for almost all the points $x$ of $S$ there exists a $j_{x} \in I$ such that $m\left(S \cap x U_{i}\right)$ and $m\left(x^{-1} U_{i} \cap S^{-1}\right)$ are both $>m\left(U_{i}\right) / 2$ as $i \geqq j_{x}$. Then $S$ is a semigroup l.a.e. If, in addition, $S$ is contained in a $\sigma$-compact subset of $G$, then $S$ is a semigroup a.e.

TheOREM 3. If $L_{S}$ is a self-adjoint vanishing algebra, then $S$ is a group l.a.e. If, in addition, $S$ is contained in a $\sigma$-compact subset of $G$, then $S$ is a group a.e.

Theorem 4. Let $L_{S}$ be a vanishing algebra. If $S$ is open, then $S$ is a semigroup l.a.e. If, in addition, $S$ is contained in a $\sigma$-compact subset of $G$, then $S$ is a semigroup a.e.

THEOREM 5. If $L_{S}$ is a maximal vanishing algebra, then $S$ is a closed

1 This research was sponsored in part by the United States Army Research Office (Durham) under contract DA-ARO(D)-31-124-G218. The author wishes to express his debt to Professor C. Ionescu Tulcea and Professor R. Ellis for many helpful discussions. 
semigroup l.a.e. If, in addition, $S$ is contained in a $\sigma$-compact subset of $G$, then $S$ is a closed semigroup a.e.

COROLLARY 3. Let $G$ be abelian and generated by some compact neighborhood of the identity element of $G$. If there exists a vanishing algebra $L_{S}$ which is a maximal subalgebra in $L^{1}(G)$, then $G$ is either the additive group of real numbers or the discrete integer group.

\section{REFERENCES}

1. P. R. Halmos, Measure theory, New York, Van Nostrand, 1950.

2. A. B. Simon, Vanishing algebras, Trans. Amer. Math. Soc. 92 (1959), 154-167.

3. - On the maximality of vanishing algebras, Amer. J. Math. 81 (1959) 613-616.

4. A. Beck, H. H. Corson and A. B. Simon, The interior points of the product of two subsets of a locally compact group, Proc. Amer. Math. Soc. 9 (1958), 648-652.

Academia Sinica, Taiwan, China and

University of Pennsyluania 